\title{
Perspectives on Life and Practice, Post COVID-19
}

As dental offices continue to face challenges and changes related to the COVID-19 pandemic, we reached out to some American Academy of Dental Sleep Medicine members to hear their perspectives moving forward. We asked them to discuss some of the continued changes they expect to see in dental sleep medicine, as well as the things they are looking forward to enjoying, post-pandemic.

\section{WHAT CHANGES IN DENTAL SLEEP MEDICINE DO YOU SEE OCCURRING AS A RESULT OF THE PANDEMIC?}

"I see more practitioners exploring how telemedicine might be a helpful tool to incorporate with patient care."

-Eric Runyon, DDS, D.ABDSM

"Prior to the pandemic, I was not using telehealth as a form of patient management and interaction. With the advent of COVID-19, telehealth became a necessity, one that I anticipate will be an integral part of how we practice dental sleep medicine moving forward."

$$
\text { -Jennifer Le, DMD, D-ABDSM, CPCC }
$$

"For patients:

1. Some COVID symptomatic patients may not be able to use oral appliances due to severity of COVID breathing problems; respiratory physician referral is mandatory.

2. Some asymptomatic, but positive individuals may develop problems: pay close attention to their complaints.

3. Expect aggravation or rise in prevalence of insomnia, sleep bruxism and pain or other conditions due to COVID stress - pay close attention.

For practice:

1. Some individuals may delay initiation of snoring and sleep apnea treatment due to COVID-related income loss. This may be counterbalanced by an increase awareness, that we have to promote, of the importance of maintaining a good general health including managing chronic snoring and sleep apnea

2. Some patients told us they are worried by the dental environment airdrops and risk of contamination: we have to do some reassurance and maybe public advertising.
Issues for further investigation, research:

1. Implementation of 'evidence based' protocol in clinic to clean oral sleep devices: how and with what? There is a lack of solid evidence. Products need to be safe for patient, our personnel and not damaging to the devices.

2. Do we know if oral breathing generates 'risk' related to micro-drops circulation in the bedroom? If yes, how can we better manage sleep oral breathing to protect sleep partner or friends (e.g., sleeping in narrow space such as when camping or boating)

3. Be aware that a SARS-CoV-2 vaccine may trigger other diseases; H1N1 vaccine adjuvant triggered narcolepsy in some individuals previously healthy."

-Gilles Lavigne, DMD, PhD

"March 16, 2020: Office shut-down - "My World" \& my "Status Quo" changed, and my life was shaken, stressed \& challenged more than I could have imagined. What now? What is next? Will there BE a "Post-COVID" practice in my future? Retire? When life presents challenges \& disruptions (PANDEMICS!) how we react is critical. This situation has caused me to really dig deep \& examine much more closely the important question: "Why do I do what I do???" I now look forward to the future, but more importantly to TODAY as we never know what tomorrow will bring (...cherish the moment...). I count my blessings more and take nothing for granted. What is my real purpose in doing what I do?

Rather than "Going through the motions" of my life \& my practice, I am now re-focusing, re-thinking, redefining my "Why." Thank you COVID (... and special thanks: Dr. J.S.) for this important awakening to continually examine the Why \& move forward with greater purpose, enthusiasm, optimism \& passion. I plan to clarify \& simplify my combined practice of dental sleep medicine \& general dentistry to be able to help more of those in need of the services I can provide. Will the crisis end? If so, there will be other challenges, but with better clarity of purpose, hurdles \& obstacles are much more easily overcome."

-David Silk, DDS, D.ABDSM

"As far as the future of DSM, it is incredibly bright! We need to be diligent in reassuring our patients that we 
are doing everything in our means to protect them and minimize their risk. As dentists we have always taken great care to sanitize our examination rooms following procedures. Now we are taking it to another level to safeguard our patients and our staffs."

-Kenneth Mogell, DMD, DABDSM

\section{WHAT ARE YOU LOOKING MOST FORWARD TO AFTER THE COVID-19 CRISIS IS OVER?}

"I am most looking forward to being able to take my 2-year-old-son on outings to all the places we haven't been able to go to, like the zoo! On a work-related answer, I am most looking forward to being able to take my staff out to dinner to thank them for their perseverance."

-Eric Runyon DDS, D.ABDSM

"The unprecedented disruption in so many aspects of our lives due to COVID-19 has me looking forward to returning to social events, such as our next Annual Meeting. I definitely miss the live presentations and the opportunities to reconnect with colleagues."

\section{-Jennifer Le, DMD, D-ABDSM, CPCC}

"Sleep is very personal for many of our patients - some of them have spent years looking for a resolution or just simply enduring their fatigue and health issues - and I can't wait until we are back running at full steam to help them find a solution."

-Kenneth Mogell, DMD, DABDSM

\section{CITATION}

Perspectives on life and practice, post COVID-19. J Dent Sleep Med. 2020;7(3).

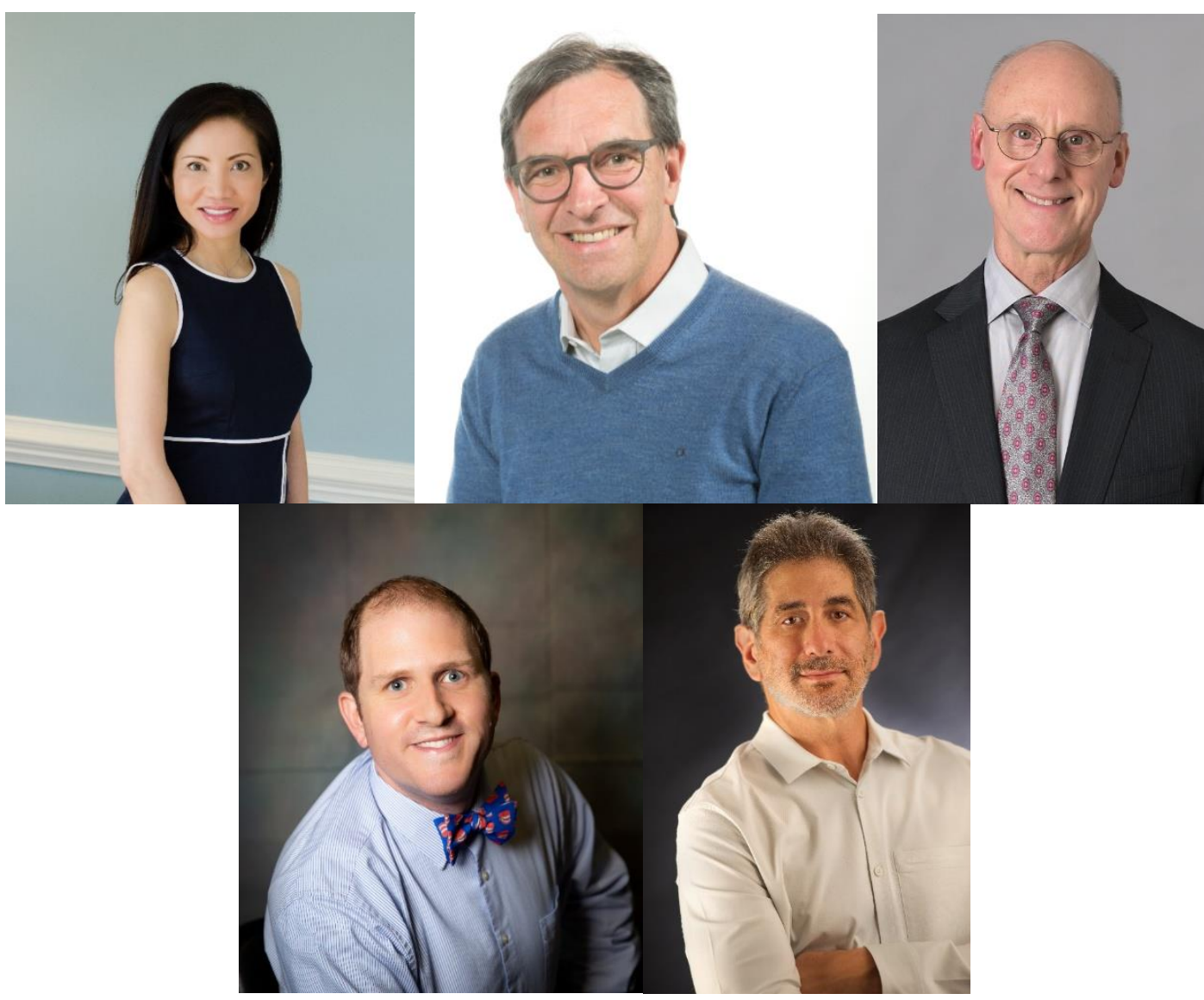

Top Row (Left to Right): Dr. Jennifer Le, Dr. Gilles Lavigne, Dr. David Silk Bottom Row (Left to Right): Dr. Eric Runyon, Dr. Kenneth Mogell 